\title{
Akupunktur in der Geburtshilfe
}

\author{
"Kinderkriegen leicht gemacht" ist \\ auf einer einschlägigen Internetseite \\ zu lesen, in der Akupunktur in der \\ Geburtshilfe angeboten wird. Die \\ Wirksamkeit der Nadelung sei für \\ diese Indikation durch verschiedene \\ Studien belegt. Wirklich?
}

- Britische Gynäkologen randomisierten 105 schwangere Frauen in fünf Gruppen. Sie erhielten während der Geburt entweder manuelle Akupunktur, Elektroakupunktur, Scheinakupunktur, Scheinelektroakupunktur oder keine Akupunktur. Alle wurden zusätzlich lege artis versorgt.

Die Ergebnisse zeigen keine Effekte der diversen Behandlungen bezüglich des Bedarfs epiduraler Analgesie, parenteraler Analgetika, der Länge der Geburt, des kindlichen Befindens oder der postpartalen Blutungen. Die Autoren schließen daraus, dass die Akupunktur in der Geburtshilfe nicht wirksam ist.

\section{Kommentar}

Die Studie ist gut gemacht und man ist somit geneigt, ihren Ergebnissen zu trauen - insbesondere auch deshalb, weil mehrere Metaanalysen die schmerzreduzierenden Effekte der Akupunktur während der Geburt nicht bestätigen konnten. Dessen unbesehen ist die Akupunktur aber weiterhin bei dieser Indikation äußerst beliebt. Wieder einmal zeigt sich also, dass in der Komplementärmedizin die Evidenz gegen Glauben und Vorurteile kaum eine Chance hat.

E. ERNST = 